\title{
A DECOMPOSITION FOR COMPLETE NORMED ABELIAN GROUPS WITH APPLICATIONS TO SPACES OF ADDITIVE SET FUNCTIONS
}

\author{
BY \\ RICHARD B. DARST
}

1. Introduction. The purpose of this paper is twofold. Our principal objective is to present a Lebesgue type decomposition Theorem (Theorem 2.3) for a generalized complete normed abelian group $G$, where generalized means (1) that the norm $(\|x\|)$ of the nonzero elements $x$ of $G$ may be infinite (i.e. if $x \in G$ and $x \neq 0$, then $0<\|x\| \leqq \infty)$ and (2) that only the subgroup of bounded elements $x$ (i.e. $[x ;\|x\|<\infty]$ ) is required to be complete. In $\S 3$, we apply this decomposition theorem to the space of finitely additive set functions on an algebra $S$ of subsets of a set $X$ in order to generalize the Lebesgue decomposition for bounded and finitely set functions on $S$ (cf. [2]).

The basic form of our decomposition depends on what we call an admissible algebra $T$ of endomorphisms on $G$ (Definition 2.3). It will be seen that $T$ is a Boolean algebra of projection operators with a condition on the manner in which projection on disjoint subgroups effects the norm. It is this latter condition which will provide our principal analytic tool.

Throughout this paper, $G$ will denote a generalized complete normed abelian group.

2. Decompositions and examples. We shall develop the notion of an admissible algebra $T$ of endomorphisms on $G$ in two stages: the first algebraic and the second analytic.

Definition 2.1. A set $T$ of endomorphisms on $G$ is said to be an algebra of endomorphisms on $G$ if whenever each of $a$ and $b$ is an element of $T$, then

(1) $a b=b a \in T$ where $a b(x)=a(b(x))$ for $x \in G$,

(2) $a a=a$, and

(3) $a^{\prime}=e-a \in T$ where $e(x)=x$ for $x \in G$.

Moreover, for each element $a$ of $T$ we let $P(a)=[x \in G ; a(x)=x]$.

We shall see that the mapping $a \rightarrow P(a)$ is an isomorphism of $T$ onto a Boolean algebra of subgroups of $G$. We have, from (2), that $\|a\|=\left\|a^{n}\right\| \leqq\|a\|^{n}$ and, hence, if $a \neq 0$ then $\|a\| \geqq 1$ ( $\|a\|$ may be infinite). Moreover, $T$ has the following properties:

(i) $0 \in T\left(a a^{\prime}=0\right)$,

(ii) $e \in T\left(e=0^{\prime}\right)$, and

(iii) $a+b-a b=\left(a^{\prime} b^{\prime}\right)^{\prime} \in T$ [note that $a^{\prime} b^{\prime}(a+b-a b)=0$ and $a^{\prime} b^{\prime}$ $\left.+(a+b-a b)=a^{\prime} b^{\prime}+\left(a b+a b^{\prime}\right)+b-a b=\left(a^{\prime} b^{\prime}+a b^{\prime}\right)+b=b^{\prime}+b=e\right]$.

Presented to the Society, April 14, 1961 under the title A Lebesgue decomposition for complete normed abelian groups; received by the editors July $17,1961$. 
Definition 2.2. If $T$ is an algebra of endomorphisms on $G$ and each of $a$ and $b$ is an element of $T$, then $a \leqq b$ means $a b=a$.

THEOREM 2.1. If each of $a$ and $b$ is an element of an algebra $T$ of endomorphisms on $G$, then

(i) $0 \leqq a \leqq e$,

(ii) $a \leqq b \Leftrightarrow a=a b \Leftrightarrow a b^{\prime}=0 \Leftrightarrow b^{\prime}=a^{\prime} b^{\prime} \Leftrightarrow a^{\prime} \geqq b^{\prime} \Leftrightarrow b=a+a^{\prime} b \Leftrightarrow$ there exists an element $c$ of $T$ such that $a=b c$,

(iii) $a b=0 \Leftrightarrow a=a b^{\prime} \Leftrightarrow a \leqq b^{\prime}$,

(iv) if $a b=0, c \leqq a$, and $d \leqq b$, then $c d=0$,

(v) $a \cap b=a b$ where $a \cap b=\sup [c \in T ; c \leqq a, c \leqq b]$,

(vi) $a \cup b=a+b-a b$ where $a \cup b=\inf [c \in T ; c \geqq a, c \geqq b]$,

(vii) $a \leqq b \Leftrightarrow P(a) \subset P(b)$,

(viii) if $a \leqq b$, then $P(b)=P(a) \oplus P\left(a^{\prime} b\right)$, and

(ix) $P(a b)=P(a) \cap P(b)$.

Proof. Parts (i), (ii), (iii), (iv), (v), and (vi) follow readily from our definitions. (vii) If $a \leqq b$, then $b^{\prime} a=a b^{\prime}=0$ and, hence, if $a x=x$, then $b^{\prime}(x)=b^{\prime} a(x)$ $=0$. (viii) It follows from (vii) that $P(a) \oplus P\left(a^{\prime} b\right) \subset P(b)$. Suppose $x \in P(b)$. Then $x=b(x)=\left(a+a^{\prime} b\right)(x)=a x+a^{\prime} b(x)$; however, $a(x) \in P(a)(a a=a)$ and $a^{\prime} b(x) \in P\left(a^{\prime} b\right)$. Thus, $x \in P(a) \oplus P\left(a^{\prime} b\right)$. (ix) We have, by (vii), that $P(a b)$ $\subset P(a) \cap P(b)$. Suppose $x \in P(a) \cap P(b)$. Then $a b(x)=a(b(x))=a(x)=x$ and, hence, $x \in P(a b)$.

We shall now introduce our analytic tool which we shall denote by Property A.

Definition 2.3. If $T$ is an algebra of endomorphisms on $G$, then $T$ is said to be an admissible algebra of endomorphisms on $G$, if $T$ has Property A: If $x \in G,\|x\|<\infty$, and $\delta>0$, then there exists $\epsilon>0$ such that if each of $a$ and $b$ is an element of $T$ and $\left\|a^{\prime} b(x)\right\|>\delta$, then $\left\|\left(a+a^{\prime} b\right)(x)\right\|>\|a(x)\|+\epsilon$.

REMARK. We note that Property $A$ is a condition only on the bounded elements of $G$. At the end of this section, we shall give examples to show (1) that $\epsilon$ may depend only on $\delta$ (Example 2.2 with $Q=1$ ), (2) that $\epsilon$ may depend on $\delta$ and $\|x\|$ but not on $x$ (Example 2.2 with $Q>1$ ), and (3) that $\epsilon$ may depend not only on $\delta$ and $\|x\|$ but also on $x$ (Example 2.4).

Henceforth $T$ shall denote an admissible algebra of endomorphisms on $G$.

THEOREM 2.2. Suppose each of $a$ and $b$ is an element of $T$, then $a \leqq b$ if and only if $\|a(x)\| \leqq\|b(x)\|$ for all $x \in G$.

Proof. If $a \leqq b$, then $b=a+a^{\prime} b$ and, hence, if $x \in G$, then $\|b(x)\|$ $=\left\|\left(a+a^{\prime} b\right)(x)\right\| \geqq\|a(x)\| ;$ in fact, inequality holds unless $\left\|a^{\prime} b(x)\right\|=0$. If $a \leq b$, then $a b^{\prime} \neq 0$ and, hence, there exists an element $x$ of $G$ such that $\left\|a b^{\prime}(x)\right\| \neq 0$. Thus, $a\left(a b^{\prime}(x)\right)=a b^{\prime}(x) \neq 0$ while $b\left(a b^{\prime}(x)\right)=0$.

CoRollary 2.2.1. If $a$ is an element of $T$ and $a \neq 0$, then $\|a\|=1$. 
Proof. We have remarked earlier that $\|a\|=\left\|a^{n}\right\| \leqq\|a\|^{n}$ and, hence, $\|a\| \geqq 1$. By Theorem 2.2, we have that $\|a\| \leqq\|e\|=1$. Thus, $\|a\|=1$.

REMARK. Later we shall give an example (Example 2.1) to show that the condition : $a \leqq b$ if and only if $\|a(x)\| \leqq\|b(x)\|$ for each $x \in G$ is not sufficient to insure a decomposition. Property $\mathrm{A}$ is equivalent to: if $x \in G,\|x\|<\infty$, and $\delta>0$, then there exists $\epsilon>0$ such that if each of $a$ and $b$ is an element of $T$, $a b=0$, and $\|b(x)\|>\delta$, then $\|(a+b)(x)\|>\|a(x)\|+\epsilon$.

LemmA 2.3.1. If $x \in G,\left\{a_{i}\right\} \downarrow$ in $T$, and $\lim _{i}\left\|a_{i}(x)\right\|<\infty$, then $\lim _{i} a_{i}(x)$ exists.

Proof. Let $L=\lim _{i}\left\|a_{i}(x)\right\|$ and let $\delta>0$. There exists a positive integer $k$ such that $\left\|a_{k}(x)\right\|<\infty$. There exists $\epsilon>0$ such that if each of $c$ and $d$ is an element of $T$ and $\left\|c^{\prime} d a_{k}(x)\right\|>\delta$, then $\left\|\left(c+c^{\prime} d\right) a_{k}(x)\right\|>\left\|c a_{k}(x)\right\|+\epsilon$. There exists a positive integer $i$ such that $i \geqq k$ and $\left\|a_{i}(x)\right\|\langle L+\epsilon$. If $j>i$, then $a_{i}=a_{j}+a_{j}^{\prime} a_{i}$. Thus, $\left\|a_{i}(x)\right\|=\left\|a_{j}+a_{j}^{\prime} a_{i}(x)\right\|<L+\epsilon \leqq\left\|a_{j}(x)\right\|+\epsilon$ and, hence, $\left\|a_{i}(x)-a_{j}(x)\right\|=\left\|a_{j}^{\prime} a_{i}(x)\right\| \leqq \delta$.

DEFinition 2.4. If each of $x$ and $y$ is an element of $G$ and $t>0$, then

(1) $Q(t, x)=[a \in T ;\|a(x)\|<t]$, and

(2) $r(t, x, y)=\sup [\|a(y)\| ; a \in Q(t, x)]$.

Lemma 2.3.2. Suppose each of $x$ and $y$ is an element of $G,\|y\|<\infty, r(t)$ $=r(t, x, y), r=\lim _{t \rightarrow 0+} r(t)<\infty$, and $\epsilon>0$. Then there exists a sequence $\left\{b_{i}\right\} \downarrow$ in $T$ such that

(1) $\lim _{i} b_{i}(x)=0$,

(2) $\lim _{i}\left\|b_{i}(y)\right\|>r-\epsilon$, and

(3) $\lim _{i} b_{i}(y)$ exists.

Proof. If $r=0$, it is sufficient to let $b_{i}=0$ for $i \geqq 1$. Suppose $r>0$ and $m$ is a positive integer such that $2^{-m}<\epsilon$. Let $t_{1}=1$. There exists $\epsilon_{1}>0$ such that

(1) $\epsilon_{1}<2^{-(m+1)}$ and

(2) if $a, b \in T$ and $\left\|a^{\prime} b(y)\right\|>2^{-(m+1)}$, then $\left\|\left(a+a^{\prime} b\right)(y)\right\|>\|a(y)\|+\epsilon_{1}$. There exists $a_{1} \in Q\left(t_{1}, x\right)$ such that $r\left(t_{1}\right)-\left\|a_{1}(y)\right\|<\epsilon_{1}$. Let $t_{2}=2^{-1}\left(t_{1}-\left\|a_{1}(x)\right\|\right)$. If $a \in Q\left(t_{2}, x\right)$, then $\left\|\left(a_{1}+a_{1}^{\prime} a\right)(x)\right\| \leqq\left\|a_{1}(x)\right\|+\|a(x)\|<t_{1}$ and, hence, $\left\|\left(a_{1}+a_{1}^{\prime} a\right)(y)\right\| \leqq r\left(t_{1}\right)<\left\|a_{1}(y)\right\|+\epsilon_{1}$. Thus, $\left\|a_{1}^{\prime} a(y)\right\| \leqq 2^{-(m+1)}$. There exists $\epsilon_{2}>0$ such that if $\left\|a^{\prime} b(y)\right\|>2^{-(m+2)}$, then $\left\|\left(a+a^{\prime} b\right)(y)\right\|>\|a(y)\|+\epsilon_{2}$. There exists $a_{2} \in Q\left(t_{2}, x\right)$ such that $r\left(t_{2}\right)<\left\|a_{2}(y)\right\|+\epsilon_{2}$. If we repeat the preceding process inductively, we obtain a sequence $\left\{a_{i}\right\}$ of elements of $T$, a sequence $\left\{e_{i}\right\}$ of positive numbers, and a sequence $\left\{t_{i}\right\}$ of positive numbers such that

(1) $t_{1}=1$ and $t_{i+1}=2^{-1}\left(t_{i}-\left\|a_{i}(x)\right\|\right)$ for $i>1$,

(2) $0<\epsilon_{i}<2^{-(m+i)}$,

(3) if $a, b \in T$ and $\left\|a^{\prime} b(y)\right\|>2^{-(m+i)}$, then $\left\|\left(a+a^{\prime} b\right)(y)\right\|>\|a(y)\|+\epsilon_{i}$,

(4) $a_{i} \in Q\left(t_{i}, x\right)$,

(5) $r\left(t_{i}\right)<\left\|a_{i}(y)\right\|+\epsilon_{i}$, and 
(6) if $a \in Q\left(t_{i+1}, x\right)$, then $\left(a_{i}+a_{i}^{\prime} a\right) \in Q\left(t_{i}, x\right)$ which implies $\left\|\left(a_{i}+a_{i}^{\prime} a\right)(y)\right\|$ $\leqq r\left(t_{i}\right)<\left\|a_{i}(y)\right\|+\epsilon_{i}$ and hence, $\left\|a_{i}^{\prime} a(y)\right\| \leqq 2^{-(m+i)}$.

For each positive integer $i, a_{i}=a_{i} a_{i-1}^{\prime}+a_{i} a_{i-1} a_{i-2}^{\prime}+\cdots+\prod_{j \leq i} a_{j}$. Let $b_{i}=\prod_{j \leq i} a_{j}$. Then $\left\{b_{i}\right\} \downarrow$ in $T$. Moreover,

$$
\begin{aligned}
\left\|b_{i}(x)\right\| \leqq & \left\|a_{i}(x)\right\| \leqq 2^{-(i-1)} \\
\left\|\left(a_{i}-b_{i}\right)(y)\right\| \leqq & \left\|a_{i} a_{i-1}^{\prime}(y)\right\|+\left\|a_{i} a_{i-1} a_{i-2}^{\prime}(y)\right\| \\
& +\cdots+\left\|\left(\prod_{1<j \leq i} a_{j}\right) a_{1}^{\prime}(y)\right\| \leqq \sum_{j<i} 2^{-(m+j)}, \quad \text { and } \\
r\left(t_{i}\right)-\left\|b_{i}(y)\right\| \leqq & r\left(t_{i}\right)-\left\|a_{i}(y)\right\|+\left\|\left(a_{i}-b_{i}\right)(y)\right\| \\
& <\epsilon_{i}+\sum_{j<i} 2^{-(m+j)}<2^{-(m+i)}+\sum_{j<i} 2^{-(m+j)}<2^{-(m)}<\epsilon .
\end{aligned}
$$

Hence, $\lim _{i}\left\|b_{i}(y)\right\| \geqq r-\epsilon$. However, $\lim _{i} b_{i}(y) \leqq \lim _{i} r\left(t_{i}\right)<\infty$ which implies (Lemma 2.3.1) that $\lim _{i} b_{i}$ exists.

Definition 2.5. If each of $x$ and $y$ is an element of $G$, then $y$ is said to be

(1) absolutely continuous with respect to $x(\bmod T)$ if for each $\epsilon>0$, there exists $\delta>0$ such that if $a$ is an element of $T$ and $\|a(x)\|<\delta$, then $\|a(y)\|<\epsilon$, and

(2) singular with respect to $x(\bmod T)$ if for each $\epsilon>0$, there exists an element $a$ of $T$ such that $\|a(x)\|<\epsilon$ and $\left\|a^{\prime}(y)\right\|<\epsilon$. Moreover, we denote by $G_{a}(x, T)$ the set of elements $h$ of $G$ which are absolutely continuous with respect to $x(\bmod T)$ and we denote by $G_{s}(x, T)$ the set of elements $u$ of $G$ which are singular with respect to $x(\bmod T)$.

LemmA 2.3.3. If $x \in G$, then each of $G_{a}(x, T)$ and $G_{s}(x, T)$ is a subgroup of Gand $G_{a}(x, T) \cap G_{s}(x, T)=0$. Moreover, if $h \in G_{a}(x, T)$, then $G_{s}(h, T) \supset G_{s}(x, T)$.

Proof. Suppose each of $y$ and $z$ is an element of $G_{a}(x, T)$ and $\epsilon>0$, then there exists $\delta>0$ such that if $a \in T$ and $\|a(x)\|<\delta$, then each of $\|a(y)\|$ and $\|a(z)\|<\epsilon / 2$ and, hence, $\|a(y+z)\|<\epsilon$. Thus, $G_{a}(x, T)$ is an algebraic subgroup of $G$. Suppose $\left\{y_{i}\right\}$ is a sequence of elements of $G_{a}(x, T), \lim _{i} y_{i}=y$, and $\epsilon>0$. Then there exists a positive integer $i$ such that $\left\|y_{i}-y\right\|<\epsilon / 2$ and there exists $\delta>0$ such that if $a \in T$ and $\|a(x)\|<\delta$, then $\left\|a\left(y_{i}\right)\right\|<\epsilon / 2$ and, hence, $\|a(y)\| \leqq\left\|a\left(y_{i}\right)\right\|+\left\|a\left(y-y_{i}\right)\right\| \leqq\left\|a\left(y_{i}\right)\right\|+\left\|y-y_{i}\right\|<\epsilon$. Thus, $y \in G_{a}(x, T)$. Suppose each of $y$ and $z$ is an element of $G_{s}(x, T)$ and $\epsilon>0$, then there exists $a$ and $b \in T$ such that $\|a(x)\|<\epsilon / 2,\|b(x)\|<\epsilon / 2,\left\|a^{\prime}(y)\right\|<\epsilon / 2$ and $\left\|b^{\prime}(z)\right\|<\epsilon / 2$ and, hence, $\|(a+b-a b)(x)\| \leqq\|a(x)\|+\|(b-a b)(x)\| \leqq\|a(x)\|+\|b(x)\|<\epsilon$ and $\left\|(a+b-a b)^{\prime}(y+z)\right\|=\left\|a^{\prime} b^{\prime}(y+z)\right\| \leqq\left\|a^{\prime}(y)\right\|+\left\|b^{\prime}(z)\right\|<\epsilon$. Suppose $\left\{y_{i}\right\}$ is a sequence of elements of $G_{s}(x, T), \lim _{i} y_{i}=y$, and $\epsilon>0$. Then there exists a positive integer $i$ such that $\left\|y_{i}-y\right\|<\epsilon / 2$ and there exists $a \in T$ such that $\|a(x)\|<\epsilon / 2$ and $\left\|a^{\prime}\left(y_{i}\right)\right\|<\epsilon / 2$ and, hence, $\left\|a^{\prime}(y)\right\| \leqq\left\|a^{\prime}\left(y_{i}\right)\right\|+\left\|a^{\prime}\left(y-y_{i}\right)\right\|$ $<\epsilon$. Therefore, $G_{s}(x, T)$ is a subgroup of $G$. Suppose $h \in G_{a}(x, T), s \in G_{s}(x, T)$, and $\epsilon>0$. Then there exists $\delta>0$ such that if $a \in T$ and $\|a(x)\|<\delta$, then 
$\|a(h)\|<\epsilon$ and, since $s \in G_{8}(x, T)$, there exists $a \in T$ such that $\|a(x)\|$ $<\min [\epsilon, \delta]$ and $\left\|a^{\prime}(s)\right\|<\min [\epsilon, \delta]$. Thus, $\|a(h)\|<\epsilon$ and $\left\|a^{\prime}(s)\right\|<\epsilon$. Hence, $s \in G_{s}(h, T)$.

REMARK. We shall give two examples (Examples 3.1 and 3.2) to show that, in general, one can not assert that $G$ is the direct sum of $G_{a}(x, T)$ and $G_{s}(x, T)$; however, Theorem 2.3 shows that $[y \in G ;\|y\|<\infty] \subset G_{a}(x, T) \oplus G_{s}(x, T)$ for each $x \in G$.

LEMMA 2.3.4. If each of $x$ and $y$ is a nonzero element of $G$, each of $\|x\|$ and $\|y\|$ is finite, and $y$ is singular with respect to $x$, then $\|x+y\|>\max [\|x\|,\|y\|]$.

Proof. Since the relation of being singular is symmetric, it is sufficient to show that $\|x+y\|>\|x\|$. There exists a sequence $\left\{a_{i}\right\}$ of elements of $T$ such that $a_{i} x \rightarrow x$ and $a_{i}^{\prime}(y) \rightarrow y$. There exists $\epsilon>0$ such that if $a \in T$ and $\left\|a^{\prime}(x+y)\right\|$ $>\|y\| / 2$, then $\|x+y\|=\left\|a(x+y)+a^{\prime}(x+y)\right\|>\|a(x+y)\|+\epsilon$. Thus, since $a_{i}(x+y) \rightarrow x$ and $\left\|a_{i}^{\prime}(x+y)\right\| \rightarrow y,\|x+y\|=\lim _{i}\left\|a_{i}(x+y)+a_{i}^{\prime}(x+y)\right\| \geqq\|x\|+\epsilon$.

LEMMA 2.3.5. Suppose each of $x$ and $y$ is an element of $G,\left\{a_{i}\right\} \downarrow$ in $T, z$ $=\lim _{i} a_{i}(y), r=\lim _{t \rightarrow 0+} r(t, x, y)$, and $\lim _{i} a_{i}(x)=0$. Then $\|z\| \leqq r$.

Proof. It is sufficient to suppose $r<\infty$. If $\epsilon>0$, then there exists $t>0$ such that if $a \in T$ and $\|a(x)\|<t$, then $\|a(y)\|<r+\epsilon / 2$ and there exists a positive integer $i$ such that $\left\|a_{i}(x)\right\|<t$ and $\left\|a_{i}(y)-z\right\|<\epsilon / 2$. Thus, $\|z\| \leqq\left\|z-a_{i}(y)\right\|$ $+\left\|a_{i}(y)\right\|<r+\epsilon$.

Theorem 2.3. Suppose each of $x$ and $y$ is an element of $G$ and $\|y\|<\infty$. Then there exists uniquely an element $h$ of $G$ and an element $s$ of $G$ such that

(1) $y=h+s$,

(2) $h$ is absolutely continuous with respect to $x(\bmod T)$, and

(3) $s$ is singular with respect to $x(\bmod T)$.

Proof. Uniqueness follows from Lemma 2.3.3; the problem is to show existence. Let $r=\lim _{t \rightarrow 0+} r(t, x, y)$. If $r=0$, let $h=y$ and $s=0\left(y \in G_{a}(x, T)\right.$ if and only if $r=0)$. Suppose $r>0$. For each positive integer $i$, there exists $\epsilon_{i}>0$ such that if each of $a$ and $b$ is an element of $T$ and $\left\|a^{\prime} b(y)\right\|>2^{-i}$, then $\left\|\left(a+a^{\prime} b\right)(y)\right\|>\|a(y)\|+\epsilon_{i}$. There exists (Lemma 2.3.2) a sequence $\{a(1, i)\} \downarrow$ in $T$ and an element $z_{1}$ of $G$ such that (1) $\lim _{i} a(1, i)(x)=0,(2) z_{1}=\lim _{i} a(1, i)(y)$, and (3) $r-\left\|z_{1}\right\|<\epsilon_{1}$. Let $y_{1}=\lim _{i} a(1, i)^{\prime}(y)=y-z_{1}$ and let $r_{1}=\lim _{t \rightarrow 0+} r\left(t, x, y_{1}\right)$. We assert that $r_{1} \leqq 2^{-1}$. Suppose, on the contrary, that $r_{1}>2^{-1}$. Then there exists a sequence $\left\{b_{i}\right\} \downarrow$ in $T$ and an element $w$ of $G$ such that (1) $\lim _{i} b_{i}(x)$ $=0$, (2) $w=\lim _{i} b_{i}\left(y_{1}\right)$ and (3) $\|w\|>2^{-1}$ (Lemma 2.3.2 again). However, $\lim _{i}\left\|b_{i}\left(y_{1}\right)\right\|=\lim _{i}\left\|b_{i} a(1, i)^{\prime}(y)\right\|$ and, hence,

$$
\begin{aligned}
\left\|z_{1}+w\right\| & =\lim _{i}\left\|a(1, i)(y)+a(1, i)^{\prime} b_{i}(y)\right\| \geqq \lim _{i}\|a(1, i)(y)\|+\epsilon_{1} \\
& =\left\|z_{1}\right\|+\epsilon_{1}>r ;
\end{aligned}
$$

but, 


$$
\lim _{i}\left\|\left(a(1, i)+a(1, i)^{\prime} b_{i}\right)(x)\right\| \leqq \lim _{i}\|a(1, i)(x)\|+\left\|b_{i}(x)\right\|=0 .
$$

This contradicts the supposition that $r_{1}>2^{-1}$. There exists a sequence $\{a(2, i)\} \downarrow$ in $T$ and an element $z_{2}$ of $G$ such that (1) $\lim _{i} a(2, i)(x)=0$, (2) $z_{2}=\lim _{i} a(2, i)\left(y_{1}\right)=\lim _{i} a(2, i) a(1, i)^{\prime}(y)$, and (3) $r_{1}-\left\|z_{2}\right\|<\epsilon_{2}$. Let $y_{2}=\lim _{i} a(2, i)^{\prime}\left(y_{1}\right)=\lim _{i} a(2, i)^{\prime} a(1, i)^{\prime}(y)$ and let $r_{2}=\lim _{t \rightarrow 0+} r\left(t, x, y_{2}\right)$. Then $r_{2} \leqq 2^{-2}$. Proceeding by induction, either there exists a smallest positive integer $i$ such that $r_{i}=0$ or $r_{i}>0$ for each positive integer $i$. In the former case we let $h=y_{i}$ and $s=\sum_{j \leq i} z_{j}$ while in the latter case we let $h=\lim _{i} y_{i}$ and $s=\sum z_{i}-$ of course, we must first show that each of $\lim _{i} y_{i}$ and $\sum z_{i}$ exists. Since $y_{i}=y-\sum_{j \leq i} z_{j}$, it is sufficient to show that $\sum z_{i}$ exists and this is done as follows. Let $s_{i}=\sum_{j \leq i} z_{j}$. If $j>i$, then $\left\|s_{j}-s_{i}\right\|=\left\|\sum_{k \leq j} z_{k}-\sum_{k \leq i} z_{k}\right\|$ $=\left\|\sum_{i<k \leq j} z_{k}\right\| \leqq \sum_{i<k \leq j}\left\|z_{k}\right\| \leqq\left(\right.$ Lemma 2.3.5) $\quad \sum_{i<k \leq j} r_{k-1} \leqq \sum_{i<k \leqq j} 2^{-(k-1)}$ $<2^{-(i-1)}$ and hence, $\lim _{i} s_{i}=\sum z_{i}$ exists. By our construction, each $z_{i} \in G_{s}(x)$ and, by Lemma 2.3.3, $G_{s}(x, T)$ is a subgroup of $G$. Thus, $s \in G_{s}(x, T)$. In order to complete a proof of Theorem 2.3, it is sufficient to show that $h \in G_{a}(x, T)$. To this end, suppose $\epsilon>0$ and $2^{-(i-1)}<\epsilon / 2$. Then $\left\|h-y_{i}\right\|$ $=\left\|s-s_{i}\right\| \leqq 2^{-(i-1)}<\epsilon / 2$ and $r_{i}=\lim _{t \rightarrow 0+} r\left(t, x, y_{i}\right) \leqq 2^{-i}<\epsilon / 2$ which implies that there exists $t>0$ such that $r\left(t, x, y_{i}\right)<\epsilon / 2$. If $a \in T$ and $\|a(x)\|<t$, then $\|a(h)\|=\left\|a\left(h-y_{i}\right)+a\left(y_{i}\right)\right\| \leqq\left\|h-y_{i}\right\|+\left\|a\left(y_{i}\right)\right\|<\epsilon / 2+\epsilon / 2=\epsilon$. Therefore, $h \in G_{a}(x, T)$.

DEFinition 2.6. The statement that a finite subset. $\left[a_{i} ; i \leqq n\right]$ of $T$ is a finite partition of $e$ in $T$ means that $a_{i} a_{j}=0$ if $i \neq j$ and $\sum_{i \leq n} a_{i}=e$.

Theorem 2.4. Suppose $x \in G,\|x\|<\infty$, and $\epsilon>0$. Then there exists a finite partition $P=\left[a_{i} ; i \leqq n\right]$ of $e$ in $T$ such that if $a \in T$ and $i \leqq n$, then at least one of $\left\|a a_{i}(x)\right\|$ and $\left\|a^{\prime} a_{i}(x)\right\|<\epsilon$.

Proof. Suppose, on the contrary, that Theorem 2.4 is false. Then there exists a pair $(x, \epsilon)$ which contradicts Theorem 2.4: $\|x\|<\infty, \epsilon>0$, and if $\left[a_{i} ; i \leqq n\right]$ is a finite partition of $e$ in $T$ then there exists an element $a$ of $T$ and a positive integer $i \leqq n$ such that each of $\left\|a a_{i}(x)\right\|$ and $\left\|a^{\prime} a_{i}(x)\right\| \geqq \epsilon$. Moreover, since the pair $(x, \epsilon)$ contradicts Theorem 2.4, for each element $a$ of $T$ at least one of the pairs $(a(x), \epsilon)$ and $\left(a^{\prime}(x), \epsilon\right)$ contradicts Theorem 2.4, i.e., if $P=\left[a_{i} ; i \leqq m\right]$ works for $a(x)$ (i.e., if $b \in T$ and $i \leqq m$ imply at least one of $\left\|b a_{i} a(x)\right\|$ and $\left.\left\|b^{\prime} a_{i} a(x)\right\|<\epsilon\right)$ and $Q=\left[b_{j} ; j \leqq n\right]$ works for $a^{\prime}(x)$, then $R=\left[a_{i} a ; i \leqq m\right] \cup\left[b_{j} a^{\prime} ; j \leqq n\right]$ works for $x$. Hence, there exists $a_{1} \in T$ such that (1) $\left\|a_{1}(x)\right\| \geqq \epsilon$ and (2) the pair $\left(a_{1}^{\prime}(x), \epsilon\right)$ contradicts Theorem 2.4; . . ; there exists $a_{i+1} \in T$ such that (1) $\left\|a_{i+1} \prod_{j \leq i} a_{j}^{\prime}(x)\right\| \geqq \epsilon$ and (2) the pair $\left(\prod_{j \leq i+1} a_{j}^{\prime}(x), \epsilon\right)$ contradicts Theorem 2.4. Let $b_{i}=\sum_{j \leq i} a_{j}$. But, by Lemma 2.3.1, $\lim _{i} b_{i}^{\prime}(x)$ exists and, hence, $\lim _{i} b_{i}(x)$ exists. This contradiction $(\|x\|<\infty)$ establishes Theorem 2.4.

We shall apply Theorem 2.4 in $\$ 3$. However, we shall first conclude this 
section by giving four examples. Our first example sheds some light on the question: How strong an analytic condition is needed on an algebra $U$ of endomorphisms on $G$ in order to assure that Theorem 2.3 will hold $(\bmod U)$ ?

EXAMPLE 2.1. In this example, $T$ will be an algebra of endomorphisms on $G$ for which Theorem 2.3 does not hold; however, $T$ will have the property that if $a, b \in T$, then $a \leqq b$ if and only if $\|a(x)\| \leqq\|b(x)\|$ for all $x \in G$.

Let $S$ be an algebra of subsets of a set $X, S$ contain an infinite number of elements, $G=[x ; x$ is a real valued function on $X,\|x\|=\sup [|x(t)| ; t \in X]]$, and $T=\left[P_{E} ; P_{E}(x)=x \cdot C(E)\right.$ where $C(E)(t)=1$ if $t \in E$ and $C(E)(t)=0$ if $t \notin E]$. Then there exist bounded elements $x$ and $y$ of $G$ such that if each of $h$ and $s$ is an element of $G, y=h+s$, and $h$ is absolutely continuous with respect to $x(\bmod T)$, then $s$ is not singular with respect to $x(\bmod T)$.

Proof. Since $S$ is infinite, there exists a sequence $\left\{E_{i}\right\}$ of non-null pairwise disjoint elements of $S$. Let $y=C(X)$ and $x=\sum 2^{-i} C\left(E_{i}\right)$. Suppose $y=h+s$ and $h \in G_{a}(x, T)$. Then there exists $\delta>0$ such that if $E \in S$ and $\left\|P_{E}(x)\right\|<\delta$, then $\left\|P_{E}(h)\right\|<2^{-1}$ and, hence, there exists a positive integer $i$ such that

$$
\left\|h \cdot C\left(\bigcup_{j>i} E_{j}\right)\right\|<2^{-1} \text {. Thus, for all } j>i, \inf \left[s(t) ; t \in E_{j}\right] \geqq 2^{-1}
$$

and, hence, $s$ is not singular with respect to $x(\bmod T)$.

ExAmple 2.2. Let $X, S, G$, and $T$ be defined as in Example 2.1 except that if $x \in G$, then $\|x\|=\left(\sum_{t \in X}|x(t)|^{Q}\right)^{1 / Q}$, where $Q$ is a real number $\geqq 1$. Then $T$ is an admissible algebra of endomorphisms on $G$.

EXAM PLE 2.3. Let $G$ be a Hilbert space, let $\left[E_{\lambda} ;-\infty \leqq \lambda \leqq \infty\right]$ be a resolution of the identity, and let $T$ be the algebra of projection operators generated by projections of the form $E_{\lambda+\mu}-E_{\lambda}, \mu \geqq 0$. Then $T$ is an admissible algebra of endomorphisms on $G$.

ExAmple 2.4. Let $X, S, G$, and $T$ be defined as in Example 2.1 except that $X$ is the set of positive integers, if $x \in G$, then $\|x\|=|x(1)|+\sum_{i \geq 1}\left(|x(2 i)|^{i}\right.$ $\left.+|x(2 i+1)|^{i}\right)^{1 / i}$, and each one element subset $[i]$ of $X$ is an element of $S$. For each positive integer $i$ we let $x_{i}=C([2 i, 2 i+1])$ and we let $a_{i}=P_{[2 i]}$. Then $\left\|x_{i}\right\|=2^{1 / i},\left\|a_{i}\left(x_{i}\right)\right\|=1$ and $\left\|a_{i}^{\prime}\left(x_{i}\right)\right\|=1$. Thus, in this example, while $T$ is admissible, the $\epsilon$ we get in satisfying Property A depends not only on $\delta$ and $\|x\|$ but also on $x$.

3. Spaces of finitely additive set functions. Throughout this section, $X$ will denote a set, $S$ will denote an algebra of subsets of $X, G$ will denote the generalized complete normed abelian group of finitely additive set functions on $S$ where the norm $(\|x\|)$ of the elements $x$ of $G$ is the total variation $(V(x, X))$ of $x$ on $X$, and $T$ will denote the admissible algebra of projection operators induced by $S$, i.e., $T=\left[P_{E} ; P_{E}(x)(F)=x(E \cap F)\right.$ for $E, F \in S$ and $x \in G]$. 
Let us recall that if $S$ is an infinite set, then there exist unbounded finitely additive set functions $x$ on $S$ (i.e., elements $x$ of $G$ such that $\|x\|=\infty$ ).

We shall extend the Lebesgue type decomposition for bounded and finitely additive set functions on a set algebra $S$ which was presented in [2]. The definitions of absolute continuity and singularity which we use here are equivalent to those which were used in [2]. In order to make this paper selfcontained with respect to notation and terminology, it is necessary to observe the following:

(1) $\left\|P_{E}(x)\right\|=V(x, E)$ for $E \in S$ and $x \in G$,

(2) $P_{E} P_{F}=P_{E \cap F}$,

(3) $P_{E}^{\prime}=P_{\left(E^{\prime}\right)}$, where $E^{\prime}=X-E$,

(4) if $E \cap F=\theta: P_{E} P_{F}=0$, then $\left\|P_{E}(x)+P_{F}(x)\right\|=\left\|P_{E \cup F}(x)\right\|=\left\|P_{E}(x)\right\|$ $+\left\|P_{F}(x)\right\|$ for all $x \in G$, and

(5) $P_{E} \leqq P_{F}$ if and only if $E \subset F$. Our first extension is the following consequence of Theorem 2.3.

THEOREM 3.1. If $x$ is a finitely additive set function on $S$ and $y$ is a bounded and finitely additive set function on $S$, then there exists uniquely an element $h$ of $G$ and an element $s$ of $G$ such that

(1) $y=h+s$,

(2) $h$ is absolutely continuous with respect to $x(\bmod T)$, and

(3) $s$ is singular with respect to $x(\bmod T)$.

THEOREM 3.2. If $x$ is a bounded finitely additive set function on $S$ and $y$ is absolutely continuous with respect to $x(\bmod T)$, then $y$ is bounded.

Proof. Since $y$ is absolutely continuous with respect to $x(\bmod T)$, there exists $\delta>0$ such that if $E \in S$ and $V(x, E)<\delta$, then $V(y, E)<1$. By Theorem 2.4 , there exists a finite partition $\left[P_{E_{i}} ; i \leqq n\right]$ of $P_{X}$ in $T$ such that if $E \in S$ and $i \leqq n$, then at least one of $V\left(x, E \cap E_{i}\right)$ and $V\left(x, E^{\prime} \cap E_{i}\right)<\delta$ and, hence, at least one of $V\left(y, E \cap E_{i}\right)$ and $V\left(y, E^{\prime} \cap E_{i}\right)<1$. For each positive integer $i \leqq n,\left|y\left(E \cap E_{i}\right)-y\left(E_{i}\right)\right|=\left|y\left(E^{\prime} \cap E_{i}\right)\right|$. Hence $\left|y\left(E \cap E_{i}\right)\right|<\left|y\left(E_{i}\right)\right|+1$ for all $E \in S$. Thus, $V\left(y, E_{i}\right) \leqq 2\left(\sup \left[\left|y\left(E \cap E_{i}\right)\right| ; E \in S\right]\right) \leqq 2\left(\left|y\left(E_{i}\right)\right|+1\right)<\infty$ for $i \leqq n$ and, hence, $\|y\|=V(y, X)=\sum_{i \leq n} V\left(y, E_{i}\right)<\infty$.

In the general setting of $\$ 2$, the analog of Theorem 3.2 is not, in general, true. For example, let $S$ be infinite and let $T^{\prime}$ be the subalgebra of $T$ which consists of 0 and $e$. Then any two nonzero elements of $G$ are absolutely continuous with respect to each other $\left(\bmod T^{\prime}\right)$; but, there exist unbounded, as well as nonzero bounded, elements of $G$.

THEOREM 3.3. If each of $x$ and $y$ is a finitely additive set function on $S$ and at least one of $x$ and $y$ is bounded, then $y$ is decomposable with respect to $x(\bmod T)$ if and only if there exists a sequence $\left\{E_{i}\right\} \downarrow$ in $S$ such that $\lim _{i} V\left(x, E_{i}\right)=0$ and $\lim _{i} V\left(y, E_{i}^{\prime}\right)<\infty$. 
Proof. If $\|y\|<\infty$ a decomposition exists; moreover, it is sufficient to let $E_{i}=\theta$ for $i \geqq 1$. Suppose $\|y\|=\infty$ and $\|x\|<\infty$.

Necessity. Suppose $y=h+s, h \in G_{a}(x, T)$, and $s \in G_{s}(x, T)$. Then, by Theorem $3.3,\|h\|<\infty$ and, by the definition of singularity, there exists a sequence $\left\{F_{i}\right\}$ of elements of $S$ such that $V\left(x, F_{i}\right)<2^{-i}$ and $V\left(y, F_{i}^{\prime}\right)<2^{-i}$. Let $E_{i}=\prod_{j \leq i} F_{j}$. Then $\left\{E_{i}\right\} \downarrow$ in $S, V\left(x, E_{i}\right)<2^{-i}$, and $V\left(y, E_{i}^{\prime}\right)=V\left(h, E_{i}^{\prime}\right)$ $+V\left(s, E_{i}^{\prime}\right) \leqq V(h, X)+\sum_{j \leq i} V\left(s, F_{j}^{\prime}\right)<\|h\|+1<\infty$.

Sufficiency. Let $y_{i}=P_{E_{i}}^{\prime}(y)$. Then $\left\|y_{i+j}-y_{i}\right\|=\left|V\left(y, E_{i+j}^{\prime}\right)-V\left(y, E_{i}^{\prime}\right)\right|$ $=V\left(y, E_{i} \cap E_{i+j}^{\prime}\right)=\left\|y_{i+j}\right\|-\left\|y_{i}\right\|$. Hence $z=\lim _{i} y_{i}$ exists and $\|z\|<\infty$; moreover, $y-z \in G_{s}(x ; T)$. By Theorem 3.1, there exist $h$ and $s_{1}$ such that $z=h+s_{1}$, $h \in G_{a}(x, T)$, and $s_{1} \in G_{s}(x, T)$. Finally, $s=y-h=(y-z)+s_{1} \in G_{s}(x, T)$.

EXAMPLE 3.1. Let $X$ be the set of positive integers and let $S$ be the algebra of all subsets of $X$. Let $x \in G$ such that if $E \subset X$ and $E \neq \theta$, then $x(E)$ $=\sum_{i \in E} 2^{-i}$. Let $y \in G$ such that $y(X)=0$ and $y([i])=1$ for all $i \in X$. Then $y$ is not decomposable with respect to $x(\bmod T)$.

EXAmpLE 3.2. Let $X$ be the half open interval $[0,1)$. Let $S$ be the algebra of subsets of $X$ generated by elements of the form $E(m, n)=\left[m / 2^{n},(m+1) / 2^{n}\right.$, $\left.0 \leqq m<2^{n}\right]$, i.e., $S=\left[\bigcup_{i \leq k} E\left(m_{i}, n_{i}\right) ; 0 \leqq m_{i}<2^{n_{i}}\right]$. We shall define $y$ inductively as follows. Let $y(X)=1, y(E(2 m, n+1))=2 y(E(m, n))$, and $y(E(2 m+1, n+1))=-y(E(m, n))$. Then $y$ is unbounded on each nonempty element of $S$. Hence $y$ is decomposable with respect to no bounded finitely additive set function on $S$ except the constant function 0 ; but, every finitely additive set function on $S$ is absolutely continuous with respect to $y$. Cameron (cf. [1]) has shown that a complex Wiener measure is unbounded on every nonempty set of the algebra on which it is defined.

Corollary 3.3.1. If $y$ is an unbounded finitely additive set function on $S$ (i.e., $y \in G$ and $\|y\|=\infty$ ), then there exists a bounded finitely additive set function $x$ on $S$ such that $y$ is not decomposable with respect to $x$.

Proof. Let $K=[E \in S ; V(y, E)<\infty]$. Then $K$ is a proper ideal in $S$. There exists a maximal proper ideal $J$ in $S$ such that $K \subset J$. There exists, uniquely, $x \in G$ such that $x(E)=0$ if $E \in J$ and $x(E)=1$ if $E \in J$. It is impossible to decompose $y$ with respect to $x$ : if $V(x, E)<1$, then $E^{\prime} \notin K$ and, hence, $V\left(y, E^{\prime}\right)$ $=\infty$.

Theorem 3.4. Suppose $S$ is a sigma algebra, $y$ is a countably additive set function on $S$, and $x$ is a finitely additive set function on $S$. Then $y$ is $(\epsilon-\delta)$ absolutely continuous with respect to $x(\bmod T)$ if and only if $y$ is $0-0 a b$ solutely continuous with respect to $x$, i.e., if and only if $E \in S$ and $V(x, E)=0$ imply $V(y, E)=0$.

Proof. Sufficiency. Suppose $y \notin G_{a}(x, T)$. Then $\lim _{t \rightarrow 0+} r(t, x, y)>0$, and, by Lemma 3.2, there exists a sequence $\left\{E_{i}\right\} \downarrow$ in $S$ such that $\lim _{i} V\left(x, E_{i}\right)=0$ 
and $\lim _{i} V\left(y, E_{i}\right)>0$. Since $S$ is a sigma algebra, $E=\cap E_{i} \in S$; moreover, $V(x, E) \leqq \lim _{i} V\left(x, E_{i}\right)=0$. Finally, since $y$ is countably additive on $S, V(y, E)$ $=\lim _{i} V\left(y, E_{i}\right)>0$.

\section{BIBLIOGRAPHY}

1. R. H. Cameron, $A$ family of integrals serving to connect the Wiener and Feynman integrals, J. Math. and Phys. 39 (1960), 126-140.

2. R. B. Darst, $A$ decomposition of finitely additive set functions, J. Math. Reine Angew. 210 (1962), 31-37.

Massachusetts Institute of Technology, Cambridge, Massachusetts

\section{ERRATA TO VOLUME 98}

C. C. Elgot. Decision problems of finite automata design and related arithmetics

Page 23, Lines 10, 11. Replace each $\hat{f}$ by $\hat{p}$.

Page 23, 3.6(b), Line 2. The words "by a finite number ..." should start a new line.

Page 24, Line 9 (second display formula). Replace “ $(a, b)$ " by “ $(b, a)$ ".

Page 46, 8.6.2, Line 5. Replace "let $n$ be the maximum" by "let $n$ be one more than the maximum".

Line 7 . Replace "for some $n$-ary $R$ " by "for some $R$ which is $n$-ary".

The third sentence (beginning on the sixth line) of $\$ 8.6 .2$ on page 46 is in error but is readily correctable. "It may be seen that $T_{m+m^{\prime}+r}^{\infty}\left(\Lambda_{x} M\right)$ $=S_{1} \cup S_{2} \cup \ldots \cup S_{k}$, where $S_{j}, j=1,2, \cdots, k$, is the set of all infinite $R_{j^{-}}$ sequences $f$ such that $(f \uparrow n) \in E_{j}$, for appropriate $R_{j}, E_{j}$, and that $k$ need not be 1 . For example, let $M$ be

$$
\begin{aligned}
& 0 \in F_{1} \wedge 0 \notin F_{2} \wedge\left(x \in F_{1} \wedge x \notin F_{2} \cdot \bigvee \cdot x \in F_{1} \wedge x \in F_{2}\right): \bigvee: \\
& 0 \notin F_{1} \wedge 0 \in F_{2} \wedge\left(x \in F_{1} \wedge x \in F_{2} \cdot \bigvee \cdot x \notin F_{1} \Lambda x \in F_{2}\right) .
\end{aligned}
$$

Then $T_{2}^{\infty}\left(\Lambda_{x} M\right)$ is the union of the set of all infinite sequences in $\langle 1,0\rangle$ and $\langle 1,1\rangle$ which begin with $\langle 1,0\rangle$ and the set of all infinite sequences in $\langle 0,1\rangle$ and $\langle 1,1\rangle$ which begin with $\langle 0,1\rangle$. Thus, in this case, $k=2$. Let $Q$ be

$\left(0 \in F_{1} \wedge 0 \notin F_{2} \cdot \bigvee \cdot 0 \notin F_{1} \wedge 0 \in F_{2}\right)$

$: \Lambda:\left(x \in F_{1} \wedge x \notin F_{2} \wedge x \in F_{3} \wedge x^{\prime} \in F_{3} \cdot \bigvee \cdot x \in F_{1} \wedge x \in F_{2} \wedge x \in F_{3} \wedge x^{\prime} \in F_{3}\right.$

- $\left.\vee \cdot x \in F_{1} \Lambda_{x \in F_{2}} \wedge x \notin F_{3} \wedge x^{\prime} \notin F_{3} \cdot \bigvee \cdot x \notin F_{1} \wedge x \in F_{2} \wedge x \notin F_{3} \wedge x^{\prime} \notin F_{3}\right)$.

Then $\Lambda_{x} M \equiv \mathrm{V}_{F_{3}} \Lambda_{x} Q$ and $T_{3}^{\infty} \Lambda_{x} Q$ is a set of $R$-sequences, for the binary $R$ indicated by the formula, beginning in a designated way and $T_{2}^{\infty}\left(\Lambda_{x} M\right)$ is a projection of $T_{3}^{\infty}\left(\Lambda_{x} Q\right)$. Quite generally it is the case that $S_{1} \cup S_{2} \cup \ldots \cup S_{k}$ is the projection of a set of $R$-sequences beginning in a designated way so 